\title{
Effect of a Traditional Chinese Medicine combined therapy on Spleen-Qi deficiency HIV/AIDS patients: a randomized controlled trial
}

\author{
Ying Liu ${ }^{1}$, Meng Sun ${ }^{2 *}$, Kelin Wang ${ }^{2}$, Gang Sun ${ }^{2}$, Xiaohong Wang ${ }^{2}$, Zhiguang Zhai ${ }^{2}$, Peizhu Sun ${ }^{1}$, Jian Wang ${ }^{1 *}$ \\ ${ }^{1}$ China Academy of Traditional Chinese Medicine, Research Center of AIDS Prevention, Beijing \\ ${ }^{2}$ China Academy of Traditional Chinese Medicine, Institute of Basic Theory, Beijing \\ *Correspondence to: Meng Sun, China Academy of Traditional Chinese Medicine, Institute of Basic Theory, Beijing, 100700; E-mail: 1368375021@qq.com \\ Jian Wang, China Academy of Traditional Chinese Medicine, Research Center of AIDS Prevention, Beijing, 100700; E-mail: 62tiger@163.com \\ Tel: 86-010-84042803; Fax: 86-010-64039230
}

Received: November 08, 2018; Accepted: November 16, 2018; Published: November 29, 2018;

\begin{abstract}
Objective: In order to observe the clinical therapeutic effects of the immune reconstitution (IR) granule on treating HIV/AIDS patients with Spleen-Qi deficiency.

Methods: One hundred Spleen-Qi deficiency HIV/AIDS patients were randomly divided into a TCM treatment group (TCMG) and a placebo-controlled group (PCG). TCMG patients were given oral administration of IR granule, in combination with Highly Active Anti-Retroviral Therapy (HAART). PCG patients were given oral administration of placebo granule in combination with HARRT therapy. Spleen-Qi deficiency symptoms of HIV/AIDS patients was determined after 6 months treatment, and patient's CD4+ and CD8+ T lymphocyte absolute count was measured after treatment. The D values ratio, average polymorphism and discreteness among TCR- receptor gene in the surface $\gamma \delta$-T cells was measured after 6 months of treatment.
\end{abstract}

Results: a 6-month cohort study of 80 outpatients with Spleen-Qi deficiency HIV/AIDS was performed. In the treated group, the results showed that the Spleen-Qi deficiency symptoms, such as weakness and fatigue, diarrhea and abnormal defecation, had been significantly improved compared to control group, the CD4+ T lymphocyte absolute count of Spleen-Qi deficiency HIV/AIDS patients present improvement, Although it showed no significant difference $(\mathrm{P}>0.05)$. It was found that in HIV/AIDS patients of Spleen-Qi deficiency, the average D value was significantly ameliorated after TCM treatment, and it suggested that the PCR products of $\gamma \delta$-T cell receptors (TCR- $\gamma$ chain) had less variation and they were closer to normal distribution through treatment.

Conclusion: In this study, we proposed the pathogenesis of Spleen-Qi deficiency in HIV/AIDS patients, revealed some of the cellular immune pathogenesis, such as attenuation of the polymorphism of $\gamma \delta$-T cell receptor genes and enhancement mucosal immune activation profile in HIV / AIDS patients of Spleen-Qi deficiency. Through the exploration, we provided a new way to evaluate the effects of TCM dialectical Therapy on the HIV / AIDS patients of Spleen-Qi deficiency, which can guide clinical treatment of Traditional Chinese Medicine to improve treatment efficacy in HIV/AIDS.

Keywords: HIV/AIDS; Spleen-Qi deficiency; T lymphocyte; $\gamma \delta$-T cell receptor genes.

\section{Introduction}

AIDS is a chronic wasting disease, accompanied by the deficiency of the body's immunity and resistance to disease [1]. In Chinese HIV/AIDS patients, the Spleen (Pi)-Qi deficiency symptom is the most proportion of Symptoms, up to $80 \%$ [2]. Usually, in theory of Traditional Chinese medicine (TCM) Spleen Qi is closely related to the immunity and body's resistance to disease, especially linked to the integrity of mucosal immunity [3]. Recent studies showed that the mucosal immune system played important roles in HIV infection and pathological progress of AIDS. Most HIV infections are transmitted through mucosa, and it was observed that HIV / AIDS carriers have defects on the mucosal immune system during the pathogenesis of HIV infection [4]. Many researches indicated that the mucosal immune system of HIV carriers were activated by destroyed gastrointestinal mucosa, inflammatory cytokines and long-term consumption of highly active antiretroviral therapy (HAART) medications [5].

Therefore in this study, With randomized double-blinded placebocontrolled method adopted, clinical observation on 80 HIV/AIDS patients of Spleen-Qi deficiency symptom treated with the immune reconstitution (IR) granule combined HARRT had been conducted. We compared the polymorphism of $\gamma \delta$-T cell receptors (TCR- $\gamma$ chain) in HIV/AIDS patients of Spleen-Qi deficiency with them after treatment, which revealed their cellular pathogenesis of mucosal immunity in Spleen-Qi deficiency HIV/AIDS patients, and provide theoretical evidence for HIV /AIDS prevention and dialectical therapy by Traditional Chinese Medicine. 


\section{Materials and Methods}

\section{Research subjects}

- Case Collection and Grouping: From September 2013 to January 2016, this study included HIV/AIDS patients from the Capital Medical University affiliated Beijing Ditan Hospital, Guangzhou Eighth People's Hospital, Yunnan traditional Chinese Medicine Research Institute. Diagnostic criteria of HIV/AIDS patients was made according to the Ministry of health "AIDS and HIV infection diagnosis standard" (WS293-2008) and "AIDS diagnosis and treatment guide (2011 Edition)" Spleenqi Deficiency Syndrome Diagnostic Criteria in TCM diagnostic criteria: According to "clinical technology solutions to treatment of AIDS using Chinese Traditional Medicine." described previously [6]. TCM diagnostic criteria: According to "clinical technology solutions to treatment of AIDS using Chinese traditional medicine." issued by the State Administration of traditional Chinese medicine of the P.R.C. in 2005. The TCM diagnostic criteria for deficiency of Spleen Qi syndrome include the primary symptoms. The primary symptoms were weakness and fatigue, food less anorexia, diarrhea and abnormal Defecation. Patients with 3 items of the primary symptoms could be determined by the Rome III diagnostic criteria.

- Inclusion Criteria: The HIV/AIDS patients were recognized as an HIV antibody positive by western blot test. The absolute count of CD4+ T cell in HIV/AIDS patients was more than 50 cells/ul, and HIV RNA below the detection value. The HIV/AIDS patients who received HAART from more than 12 months to less than 24 months was less than 200 cells/ul, and who received HAART for more than 24 months was less than 350 cells/ul. All HIV/AIDS patients voluntarily participated in this study and signed the informed consent form. Standards of syndrome differentiation were based on "Clinical Technology Solutions of National Free TCM HIV/AIDS Treatment.

- Exclusion criteria: Before entering the group, patients with serious opportunistic infection which not been controlled; Patients who are participating in clinical trials of other drugs; Patients with autoimmune diseases; Pregnant or lactating women, or ready to pregnant women; Any patient with renal insufficiency; patients with mental or language barriers, cannot fully understand the content of the test or to give good cooperation.

\section{Randomization, double-blind method}

- This trial was adopted randomized double blinded placebo parallel controlled method, and was registered in the Chinese clinical trial registry, the registration number: ChiCTR-TRC-13003716. One hundred HIV/AIDS cases enrolled in this study were randomized into two groups. The whole observation course was 6 months. Statistical professionals using SAS software to generate random number table, a random process was achieved by clinical evaluation center of China Academy of Traditional Chinese Medicine. After determining the inclusion and exclusion criteria of the subjects, the researcher obtained the "subjects number" and "drug number" through the central randomization system.

\section{Therapy}

- One hundred HIV/AIDS cases enrolled in the study were randomized into two groups, The treatment group with 50 cases received IR granule +HAART $(\mathrm{d} 4 \mathrm{~T}+\mathrm{ddI}+\mathrm{NVP})$, while the placebo group with 50 cases received placebo + HAART $(\mathrm{d} 4 \mathrm{~T}+\mathrm{ddI}$ $+\mathrm{NVP}$ ); the treatment last for 6 months in either groups.

- The IRgranule were provided by Jiangyin Tian Jiang Pharmaceutical Co., Ltd. Specification: 5.5g/bag; Lot number: 1311327; The recipe consisted of Huangqi (Astragalus membranaceus), Dangshen (Codonopsis Pilosula), Lingzhi (Ganoderma Lucidum), Gouqizi (Fruit of Chinese wolfberry), Bajitian (Morinda Officinalis). The placebo is designed and manufactured by the above manufacturers. It was taken orally twice a day.

- In the course of the study, 80 subjects completed the trial, in which include the treatment group 40 cases and the placebo group 40 cases. The treatment group: $(43.64+11.2)$ years old, Height $(165.87+7.52) \mathrm{cm}$, weight $(58.56+8.41) \mathrm{kg}$; the placebo group: $(43.92 \pm 10.54)$ years old, Height $(166.77 \pm 7.12) \mathrm{cm}$, and weight $(59.89 \pm 9.35) \mathrm{kg}$. Immune indicators (The absolute count of CD4+ and CD8+ T cell), body temperature, heart rate, respiration, blood pressure, the baseline of symptoms and signs had no significant difference between the two groups.

\section{Research methods}

- $2 \mathrm{ml}$ peripheral venous blood was collected in the morning, Mix the blood samples well in the blood collection tubes. Take 300ul anti-coagulated whole blood. DNA samples from whole blood cell were extracted with Promega Maxwell 16 Blood DNA Purification Kit. Protein concentrations in all samples were confirmed to meet the requirementsof fluorescentPCR (OD 260/280 values between 1.8and 2.0). CD4+an CD8+ T lymphocyte absolute count in patients and other relevant information were described previously [7].

- Fluorescent PCR The primers were synthesized by Shanghai Shenggong bioengineer company. PCR amplifications were carried out in two tubes. Primersets for TCR- $\gamma$ chain and PCR program were described previously [8]. After PCR amplification, fluorescent PCR products were analyzed with ABI -3100 gene fragment analyzer and GeneMarker V1.95 scan analysis software. The area of Mean values, standard deviations, and Distances to mean ( $D$ value) of TCR- $\gamma$ chain distribution in samples and controls were recorded.

\section{Medical ethics}

In accordance with the Helsinki declaration, all patients signed informed consent, And a comprehensive understanding of the purpose, procedures, possible risks and benefits of this study before participating in the trial approved by China Academy of Traditional Chinese Medicine ethics committee.

\section{Statistical analysis}

All analyses were performed using SPSS software version 17.0. Data are shown with mean \pm standard deviation. An independent sample 
t-test was used for quantitative data fitting a normal distribution. A paired sample t-test was used to compare the data from the two groups before and after treatment. $\mathrm{P}<0.05$ was considered as a statistically significant difference.

\section{Results and Discussion}

After 6 months of treatment, in the treated group, the results showed that the Spleen-Qi deficiency symptoms, such as weakness and fatigue, food less anorexia, diarrhea and abnormal Defecation, had been significantly improved, which compared to that of the control (Table 1).

Table 1. The score of Spleen-Qi deficiency symptoms before and after treatment

\begin{tabular}{|l|c|c|c|c|}
\hline & \multicolumn{2}{|c|}{ Treatment group } & \multicolumn{2}{c|}{ Control group } \\
\hline & Before & After & Before & After \\
\hline $\begin{array}{l}\text { weakness and } \\
\text { fatigue }\end{array}$ & $2.08 \pm 0.27$ & $0.86 \pm 0.58^{\mathrm{ab}}$ & $2.06 \pm 0.38$ & $1.25 \pm 0.35^{\mathrm{a}}$ \\
\hline food less anorexia & $2.13 \pm 0.45$ & $1.31 \pm 0.38^{\mathrm{a}}$ & $2.11 \pm 0.41$ & $1.35 \pm 0.35^{\mathrm{a}}$ \\
\hline diarrhea & $2.15 \pm 0.46$ & $0.89 \pm 0.39^{\mathrm{ab}}$ & $1.96 \pm 0.45$ & $1.33 \pm 0.50^{\mathrm{a}}$ \\
\hline
\end{tabular}

Data were shown as mean \pm SD $(n=40)$. Statistical comparisons were made by the paired sample t-test. ${ }^{\mathrm{a}}$ indicates $\mathrm{P}<0.05$, compared to that of before; ${ }^{\mathrm{b}}$ indicates $\mathrm{P}<0.05$, compared to that of control.

In the treated group, $\mathrm{CD} 4+$ count increased on average, while in the control group it decreased on Average. Although it showed no significant difference as compared with before treatment, while CD8+ count in the IR granule group and control group presented an elevating rather than a decreasing trend (Table 2).

Table 2. Comparison of CD4+ and CD8+ Count between the two groups after 6 months of treatment

\begin{tabular}{|c|c|c|c|c|}
\hline & \multicolumn{2}{|c|}{ Treatment group } & \multicolumn{2}{c|}{ Control group } \\
\hline & Before & After & Before & After \\
\hline CD4+ & $209.12 \pm 72.09$ & $225.45 \pm 76.23$ & $212.78 \pm 79.01$ & $211.11 \pm 80.45$ \\
\hline CD8+ & $658.09 \pm 251.12$ & $632.65 \pm 263.17$ & $787.64 \pm 441.98$ & $736.52 \pm 350.64$ \\
\hline
\end{tabular}

Data were shown as mean $\pm S D(n=40)$. Statistical comparisons were made by the paired sample t-test

Compared to control group, the average D value of $\gamma \delta$-T cell receptor genes (TCR- $\gamma \mathrm{f} 2$ chain) in Spleen-Qi deficiency HIV/AIDS patients was significantly ameliorated after treatment. The discreteness of TCR- $\gamma$ f1 chain did not show significant difference, yet indicating greater polymorphism after treatment (figure 1). And it suggested that the PCR products of $\gamma \delta$-T cell receptors (TCR- $\gamma$ chain) in Spleen-Qi deficiency HIV/AIDS patients had less variation and they were closer to normal distribution through TCM dialectical therapy.

Defensing, one major function of spleen as stated in Miraculous Pivot (Lingshu), is closely associated with its immune function in Western Medicine. A famous saying, quoted from Master Zhang Zhongjing, that a healthy spleen can keep one from evils of the four seasons, explicitly points out the significance of spleen in human defensing function. Qi and blood transformation and production insufficiency due to the spleen deficiency could lead to weakening of human defensing strength. The rules of distribution and development of Traditional Chinese Medicine syndromes of HIV/AIDS cases was reported by Our research Team, the disease location of HIV infector is spleen, main syndrome is deficiency of Spleen Qi [9]. Therefore, strengthening Spleen Qi is a fundamental TCM treatment therapy. For example, our research Team reported that one randomized, doubleblinded, placebo-controlled clinical trial tested Aining granule plus HAART on 100 HIV/AIDS cases [10]. Aining granule consists of Astragali mongolici, Fructus lycii, Panax notoginseng, Poria cocos, and Radixet Rhizoma glycyrrhizae, which can tonify qi and invigorate the spleen. Although there was no significant difference between the two groups in viral load after treatment, CD4 + T counts declined less in the Aining group than in the control group. The treatment group showed significant improvement of symptoms such as fatigue, anorexia, and diarrhea. Meanwhile, Yi Ai Kang capsule, as a Chinese hospital herbal preparation, has been used to treat HIV/AIDS in Henan Province, and more than 4,500 infectors take this preparation every day [11]. Its main ingredients include Astragalus membranaceus, ginseng, Ligusticum chuanxiong, which can strengthens the Spleen Qi, invigorates vital energy. The treatment was effective in improving Spleen-Qi deficiency symptoms, such as reducing fatigue and spontaneous perspiration.

The purpose of TCM is to maintain the Spleen Qi and body resistance so as to arrest the progressing of the disease. If someone has enough strong Spleen Qi and body immunity through TCM, even if infected with HIV, he can live with it, being a long term HIV carrier, so as to postpone onset and death [12].

Current idea of Western Medicine to cure HIV/AIDS patients is firstly against for HIV virus (such as HAART therapy can suppress HIV replication), though HAART therapy can significantly reduce plasma viral load and reduce the morbidity and mortality of AIDS, but it is difficult to remove virus lurking in the cell, and long-term HAART therapy of HIV infection in asymptomatic people will directly impair the infector's gastrointestinal mucosa, led to digestive discomfort (including diarrhea, abdominal distension) [13]. While Traditional Chinese Medicine (TCM) attaches great importance to regulate Spleen Qi and enhance the body's resistance to disease. It was found that a lot of Chinese medicine could improve the gastrointestinal mucosa, strong Spleen-Qi and body immunity [14]. For example, it was reported Wendan granule can reduce the incidence of adverse symptoms caused by HARRT, especially gastrointestinal adverse symptoms [15]. In this study, the IR immune granule consists of Huangqi (Astragalus membranaceus), Dangshen (Codonopsis Pilosula), Lingzhi (Ganoderma Lucidum), Gouqizi (Fruit of Chinese wolfberry), Bajitian (Morinda Officinalis). It tonifies Spleen Qi, nourishes yin-yang, clears detoxifies. It was demonstrated that the IR granule plus HAART can improve the Spleen-Qi deficiency symptoms, such as weakness, diarrhea and abnormal Defecation, compared to the control group.

Now the research of immune reconstitution (IR) of HIV/AIDS patients is gradually paid more attention, but the indicators of clinical evaluation of Traditional Chinese Medicine (TCM) treatment's efficacy focus more on HIV/AIDS patients' clinical complaints, such as abnormal symptoms and their immune system function are still 
mainly based on CD4+ T lymphocyte count and viral load, which become the key problems to be concerned as soon as possible [16]. Although CD4 + T cell counts and HIV loads are gold standard evaluation criteria for AIDS therapy as recommended by the World Health Organization (WHO), CD4+ T lymphocyte count cannot fully reflect the functions of the immune system, such as the integrity of mucosal immunity; on the other hand, viral load measurement is not suitable for evaluation of TCM treatment's efficacy on HIV/AIDS patients [17]. Studies have shown that the pathogenesis of AIDS that HIV virus directly killing CD4+ T cells in the body caused the decline in CD4+ T cells and thus triggered AIDS remains to be questionable, the degree of immune activation in HIV/AIDS patients can predict the progression of the disease better than viral load in plasma [18]. Recent studies showed that the mucosal immune system played important roles in HIV infection and pathological progress of AIDS [19]. Most HIV infections are transmitted through mucosa, and it was observed that HIV / AIDS carriers have defects on the mucosal immune system during the pathogenesis of HIV infection. Many researches indicated that the mucosal immune system of HIV carriers were activated by destroyed gastrointestinal mucosa, inflammatory cytokines and longterm consumption of highly active antiretroviral therapy (HAART) medications [20].

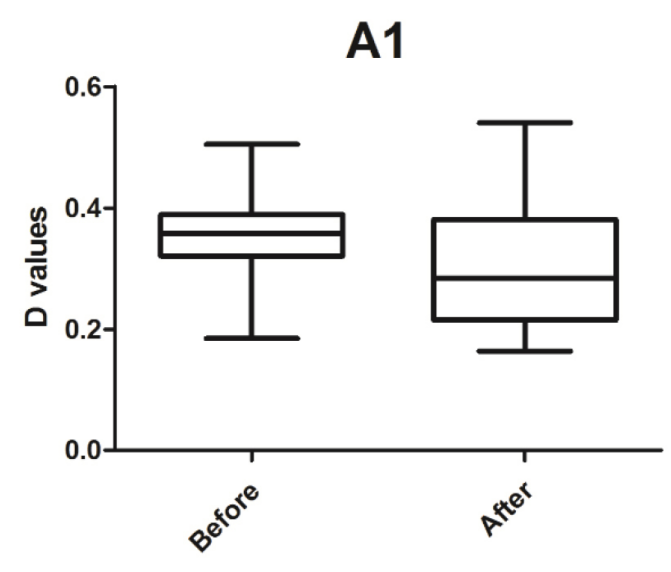

Treatment group

B1

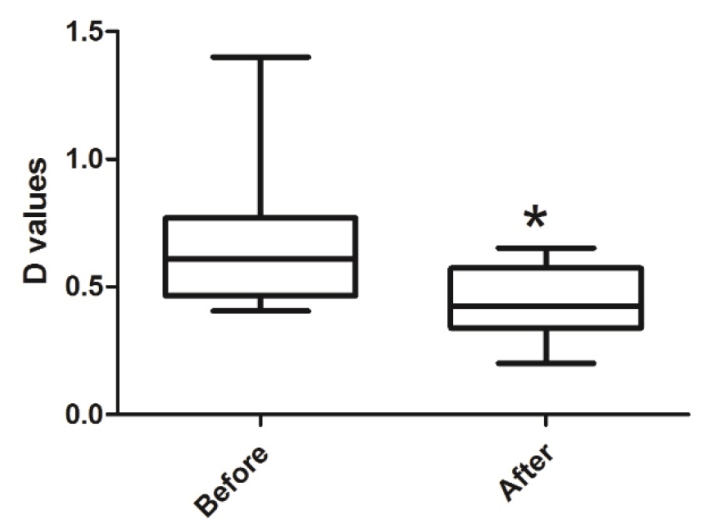

Treatment group

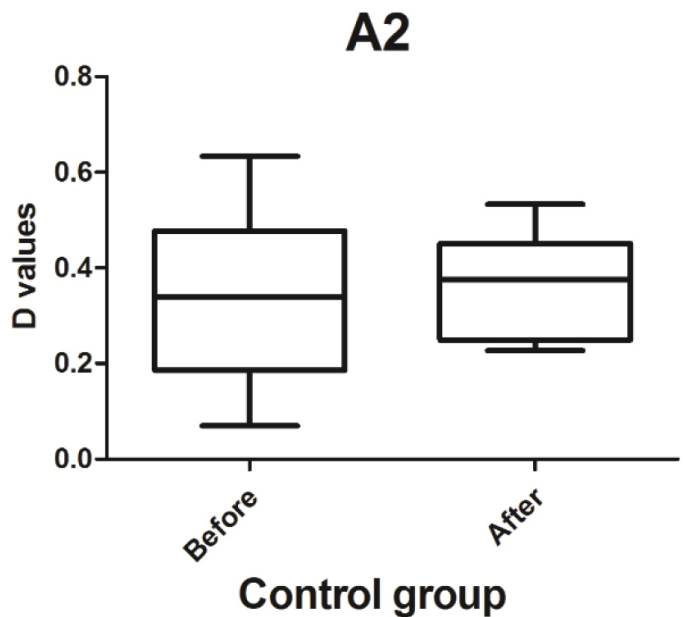

B2

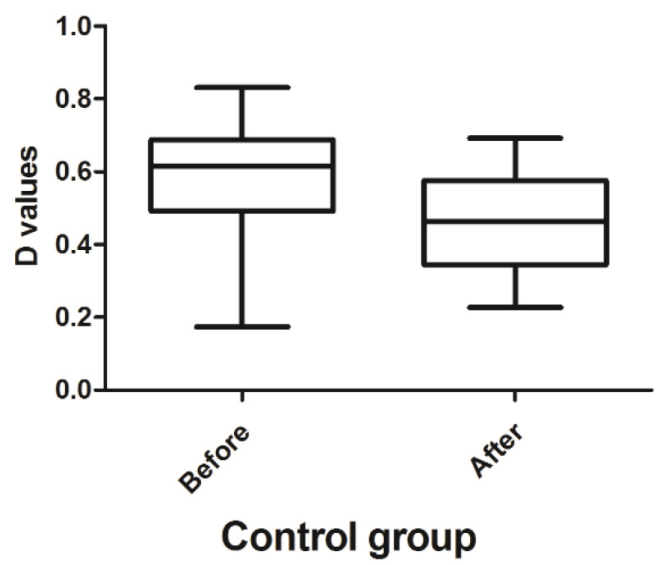

Figure 1. Comparison of gene polymorphism among $\gamma \delta$-T cell receptors (TCR- $\gamma$ chain) between the two groups after 6 months of treatment. D values of TCR- $\gamma$ f1 chain in Treatment group (A1) and Control group (A2). D values of TCR- $\gamma$ f2 chain in Treatment group (B1) and Control group (B2). *indicates $\mathrm{P}<0.05$, compared to that of before.

Recently, more and more reports have uncovered the associations between mucosal immune function and Spleen-Qi deficiency [21, 22]. It was summarized that immune activation mediators involved in the animal models of Spleen-Qi deficiency and found out that cytokines, inflammation in gastrointestinal mucosa were all closely related to the syndrome of Spleen-Qi deficiency, which directly certified the tight connection between mucosal immune function and Spleen-Qi deficiency [23]. In this research, a 6-month cohort study of 80 outpatients with Spleen-Qi deficiency HIV/AIDS tested the IR granule's effects on regulation of immune activation molecules 
$\gamma \delta$-T cell receptors related to mucosal immune activation. The results suggested that one potential mechanism of action for the IR granule may lie in its ability to up-regulate polymorphism levels on $\gamma \delta$-T cell receptors, thereby modulating immune activation of $\gamma \delta$-T cell. Although $\gamma \delta$-T cells do not have CD4 receptors and HIV virus does not infect and damage them directly, they are critical in regulating mucosal immune system. Since they mainly exist on mucosal membranes, they are considered as the first line of defense when attacked by pathogens [24]. For example, $\gamma \delta$-T cell impairment in early stage of HIV-1 infection gets more and more attention, in the early stage of infection, the count of $\gamma \delta$-T cells decreased dramatically [25]. It has been reported that $\gamma \delta$-T cells could be marker cells during HIV infection and AIDS progress [26].

Theoretically, without specific stimulation of foreign antigens, TCR- $\gamma$ chain gene fragments of $\gamma \delta$-T receptors recombinate randomly. Correspondingly, the TCR- $\gamma$ chain gene fragment scans presented a typical bell-shaped Gaussian distribution in healthy individuale. Undamaged polymorphism of healthy $\gamma \delta$-T cell receptors ensures their potentials of recognizing various foreign antigens. In cases of activated immune system, such as in virus infected and lymphocytoma patients, TCR genes of $\gamma \delta$-T cell receptors may exhibit specific single combination and expression. Once the polymorphism of the surface receptor genes is damaged, the probability of being infected by other pathogens increases correspondingly. Recently, the changes of $\gamma \delta$-T cells in HIV-infected peripheral blood have been reported. Our post study revealed that TCR- $\gamma$ chain genes of $\gamma \delta$-T cell receptors in HIV/AIDS patients exhibited single recombination, impaired polymorphism, lowered complexity and increased system entropy [8]. The numbers and functions of $\gamma \delta$-T cells remain normal in HIV-1 infected patients without long-term non-progressor HAART therapy (natural viral suppressors, NVSs), while those of other HIV infected individuals couldn't restore to the normal level even after HARRT treatment $[27,28]$.

From this point of view, it is worth knowing whether certain Chinese herbs, which directly regulate the functions of digestive mucosa and improve the Spleen-Qi deficiency symptoms, could help to recover $\gamma \delta$-T cell functions in HIV/AIDS patients of Spleen-Qi defiency. In this study, to some extent our results verified the immune activation state of $\gamma \delta$-T cell was improved in the Spleen-Qi deficiency HIV/AIDS patients through the treatment of the IR granule.

\section{Conclusion}

Currently, the research on the mechanism on TCM dialectical therapy among HIV/AIDS patients of Spleen-Qi deficiency is absent. In this study, with randomized, double-blind, controlled method and multiple-primer fluorescence PCR fragment analysis, gene recombination of $\gamma \delta$-T cell receptors (TCR- $\gamma$ chain) following treatment of the IR granule was detected. Compared with control group, the study showed that the polymorphism of $\gamma \delta$-T cell receptor genes appeared recovered, and the mucosal immune activation modulated by $\gamma \delta$-T cell receptor genes in HIV / AIDS patients of of Spleen-Qi deficiency presented obviously improvement. This study provides a new way to evaluate the effects of TCM dialectical Therapy on the HIV / AIDS patients of Spleen-Qi deficiency.

\section{Acknowledgments}

This work was supported by the National Major Science and Technology Specific Project of China (No.2013zx10005001) and National Natural Science Foundation of China (No. 81573816) and National Natural Science Foundation of China (No. 81403271)

\section{References}

1. Killian MS, Levy JA (2011) HIV/AIDS: 30 years of progress and future challenges. Eur J Immunol 12(41): 3401-11. [Crossref]

2. Liu Y, Zou W,. Xian QF, et al. (2013) Four hundred and eighty five cases of HIV/ AIDS patients based on expert interpretation of characteristics of syndromes. Zhongguo Zhong Yao Za Zhi 38(15): 2468-71. [Crossref]

3. Wang CF, Li Z, Xu LR. (2012) Discuss the spleen deficiency of AIDS pathogenesis from the point of view intestinal mucosal immune system damage. CJTCMP, 27(1): $72-74$.

4. Cao W, Mehraj V, Vyboh K, Li T, Routy JP. (2015) Antiretroviral therapy in primary HIV-1 Infection: Influences on Immune Activation and Gut Mucosal Barrier Dysfunction. AIDS Rev. 17(3): 135-46. [Crossref]

5. George MD, Asmuth DM. (2014) Mucosal immunity in HIV infection: what can be done to restore gastrointestinal-associated lymphoid tissue function? Curr Opin Infect Dis. 27(3): 275-81. [Crossref]

6. Jiang FL, Fu C, Ma JP, et al. (2011)Traditional Chinese medicine syndrome factors of patients with HIV infection or AIDS in China. Zhong Xi Yi Jie He Xue Bao 9(9): 955-64. [Crossref]

7. $\mathrm{Xu}$ JQ, Wang JJ, Han LF, et al. (2006) Epidemiology, clinical and laboratory characteristics of currently alive HIV-1 infected former blood donors naive to antiretroviral therapy in Anhui Province, China.Chinese medical journal 119(23): 1941-8. [Crossref]

8. Sun M, Liu Y, Wang KL, et al. (2014) Characteristics study on the diversity of $\gamma \delta$-T lymphocyte receptor gene in the HIV/AIDS patients. Chinese Journal of Basic Medicine in Traditional Chinese Medicine. 20(12): 1661-1664.

9. Liu Y, Wang Y, Zou W, Wang J. (2010) Chinese Medical clinical research Analysis of HIV /AIDS, Chinese Journal of Basic Medicine in Traditional Chinese Medicine. 161(1): 80-81.

10. Wang J, Liu Y, Zou W, He YL, Yan SY, Yuan YH. (2008) Clinical observations on $100 \mathrm{HIV} / \mathrm{AIDS}$ cases treated with Chinese herb Aining Granule plus HAART. Chin J AIDS STD (Chin). 14(2): 101-7.

11. Xu LR, Guo HJ, Liu ZB, Li Q, Yang JP, He Y. (2015) Unified-Planning, gradedadministration, and centralized controlling: a management modality for treating acquired immune deficiency syndrome with Chinese Medicine in Henan Province of China. Chin J Integr Med. 21: 243-8. [Crossref]

12. Yang FZ, Yan JH, Wang J. (2005) The research on the pathogenesis of AIDS in Spleen-Qi deficiency in TCM. Chinese Journal of Basic Medicine in Traditional Chinese Medicine. 11(2): 147-148.

13. Cai J, Xiao J, Zhang Q et al. (2014) Side effects and tolerability of post-exposure prophylaxis with zidovudine, lamivudine, and lopinavir/ritonavir: a comparative study with HIV/AIDS patients. Chinese medical journal (Engl) 127(14): 2632-6. [Crossref]

14. Liu ZB, Yang JP and Xu LR. (2015) Effectiveness and safety of traditional Chinese medicine in treating acquired immune deficiency syndrome: 2004-2014. Infectious Diseases of Poverty. 4: 59. [Crossref]

15. Zhang XW, Guo HJ, Jiang F, Chen XM. (2012) Study on Wendan granule in treatment of gastrointestinal adverse caused by antiretroviral therapy. Chin J Exp Tradit Med Form (Chin). 18(7): 252-4.

16. Wang J, Zou W, Liu Y. (2013) Analysis on efficacy action points of HIV/ AIDS treatment with traditional Chinese medicine and antiretroviral drugs. Zhongguo Zhong Yao Za Zhi 38(15): 2504-6. [Crossref]

17. Wang K L. (2006) The sense of reduction of AIDS mortality in China by early intervention. Journal of Public Health and Preventive Medicine 17(5): 118.

18. Brenchley JM, Price DA, Schacker TW, et al. (2006) Microbial transloction is a cause of systemic immune activation in chronic HIV infection. Nat Med 12: 13651371. [Crossref]

19. Mudd JC, Brenchley JM. (2016) Gut Mucosal Barrier Dysfunction, Microbial Dysbiosis, and Their Role in HIV-1 Disease Progression. J Infect Dis. $214 \mathrm{Suppl}$ 2: S58-66. [Crossref]

20. Torres RA, Lewis W. (2014) Aging and HIV/AIDS: pathogenetic role of therapeutic side effects. Lab Invest 94(2): 120-8. [Crossref]

21. Wang FY, Su M, Zheng YQ, et al. (2015) Herbal prescription Chang'an II repairs intestinal mucosal barrier in rats with post-inflammation irritable bowel syndrome. Acta Pharmacol Sin. 36(6): 708-15. [Crossref] 
Meng Sun and Jian Wang (2018) Effect of a Traditional Chinese Medicine combined therapy on Spleen-Qi deficiency HIV/AIDS patients: a randomized controlled trial

22. Liu F, Liu Y, Tian C. (2015) Effect of Rhizoma Atractylodis extract in protecting gastric mucosa and modulating gastrointestinalimmune function in a rat model of spleen deficiency. Nan Fang Yi Ke Da Xue Xue Bao. 35(3): 343-7, 354 [Crossref]

23. Yu B, Zhou C, Zhang J, Ling Y, Hu Q, Wang Y, Bai K. (2014) Latest Study on the Relationship between Pathological Process of Inflammatory Injury and the Syndrome of Spleen Deficiency and Fluid Retention in Alzheimer's Disease. Evid Based Complement Alternat Med. 2014: 743541. [Crossref]

24. Cimini E, Agrati C, D’Offizi G, et al. (2015) Primary and Chronic HIV Infection Differently Modulates Mucosal V $\delta 1$ and V $\delta 2$ T-Cells Differentiation Profile and Effector Functions. PLoS One. 10(6): e0129771.
25. Espíndola MS, Soares LS, Galvão-Lima LJ, et al. (2016) HIV infection: focus on the innate immune cells. Immunol Res. 64(5-6): 1118-1132 [Crossref]

26. Pauza CD, Poonia B, Li H, Cairo C, Chaudhry S. (2015) $\gamma \delta$ T Cells in HIV Disease: Past, Present, and Future. Front Immunol. 30 (5): 687. [Crossref]

27. David J. Riedel DJ, Sajadi MM, et al. (2009) Natural viral suppressors of HIV-1 have a unique capacity to maintain $\gamma \delta$ T cells. AIDS, 23(15): 1955-1964. [Crossref]

28. Andrew M. Hebbeler AM, Propp N, Cairo C, et al. (2008) Failure to Restore the V $\gamma 2-\mathrm{J} \gamma$ 1.2 Repertoire in HIV-infected Men Receiving Highly Active Antiretroviral Therapy ( HAART). Clin Immunol, 128(3): 349-357. [Crossref]

\section{Citation:}

Ying Liu, Meng Sun, Kelin Wang, Gang Sun, Xiaohong Wang, Zhiguang Zhai, Peizhu Sun, Jian Wang (2018) Effect of a Traditional Chinese Medicine combined therapy on Spleen-Qi deficiency HIV/AIDS patients: a randomized controlled trial. J Clin Res Med Volume 1(3): 1-6. 